\title{
Mitral valve Bioprosthesis is the ideal choice for younger women population
}

\author{
hamdy singab ${ }^{1}$ and Gamal Sami ${ }^{2}$ \\ ${ }^{1}$ Ain Shams University Faculty of Medicine \\ ${ }^{2}$ Nasser Institute for Research and Treatment
}

May 14, 2020

\begin{abstract}
Background: The ultimate goal of mitral valve surgery in younger women is to extend life expectancy and improve quality of life. Mitral valve replacement prosthesis in the middle-aged women is a difficult choice between the lifelong anticoagulation of a mechanical prosthesis versus a limited long-term durability of the bioprosthesis. The current trend towards reducing the women age for selection of the mitral bioprosthesis over a mechanical prosthesis lead to a dilemma for younger women decision making.1,2 The aim of this study is to compare the clinical out-comes after mitral valve bioprosthesis versus mechanical prosthesis in younger women population when mitral valves repair not feasible or unsuitable. Methods: A single-centre prospective non randomized registration of all women patients undergoing Mitral Valve Replacement at our center from January 2010 to February 2020 established. Results: Between January 2010 and February 2020, 355 female patients underwent mitral valve replacement at our center, 174 female patients of them had mitral valve replacement with mitral bioprosthesis (Mb) and 181 female patients of them had mitral valve replacement with mitral mechanical $(\mathrm{Mm})$ prosthesis. Our study proved that the use of anticoagulation among the Mm young women associated with a remarkable risk of postoperative bleeding, abortion and increase frequency of the pregnancy related complication of the anticoagulation $(\mathrm{p}=<0.0001)$, moreover, there is a considerable survival benefits for younger women patients received $\mathrm{Mb}(\mathrm{p}=0.0001)$. Conclusions: These data confirm that the impulse for the use of mitral bioprosthesis for young women population has been great with an astonishing survival benefits.
\end{abstract}

\section{Mitral valve Bioprosthesis is the ideal choice for younger women population}

Hamdy singab ${ }^{1 *}$, PhD. Gamal sami², PhD.

${ }^{1}$ Department of Cardiovascular and thoracic Surgery, Faculty of medicine, Ain Shams University, Cairo, Egypt.

2 1.Nasser Institute for Research and Treatment, Cardiothoracic Surgery Cairo, Egypt.2.Department of Cardiovascular and thoracic Surgery, Faculty of medicine, Ain Shams University, Cairo, Egypt.

*Corresponding author:

Department of Cardiac Surgery,

Faculty of medicine, Ain Shams University,

Abbasia square, Cairo, Egypt,

P.O. Box 11517, Cairo, Egypt

Telephone: 00201001008859

Fax: 002022604465 
E-mail: hamdi_singab@hotmail.com

drhamdy-ahmed@med.asu.edu.eg

\section{Abstract \\ Background:}

The ultimate goal of mitral valve surgery in younger women is to extend life expectancy and improve quality of life. Mitral valve replacement prosthesis in the middle-aged women is a difficult choice between the lifelong anticoagulation of a mechanical prosthesis versus a limited long-term durability of the bioprosthesis. The current trend towards reducing the women age for selection of the mitral bioprosthesis over a mechanical prosthesis lead to a dilemma for younger women decision making. ${ }^{1,2}$

The aim of this study is to compare the clinical out-comes after mitral valve bioprosthesis versus mechanical prosthesis in younger women population when mitral valves repair not feasible or unsuitable.

\section{Methods:}

A single-centre prospective non randomized registration of all women patients undergoing Mitral Valve Replacement at our center from January 2010 to February 2020 established.

\section{Results:}

Between January 2010 and February 2020, 355 female patients underwent mitral valve replacement at our center, 174 female patients of them had mitral valve replacement with mitral bioprosthesis (Mb) and 181 female patients of them had mitral valve replacement with mitral mechanical (Mm) prosthesis. Our study proved that the use of anticoagulation among the Mm young women associated with a remarkable risk of postoperative bleeding, abortion and increase frequency of the pregnancy related complication of the anticoagulation $(p=<0.0001)$, moreover, there is a considerable survival benefits for younger women patients received $\mathrm{Mb}(p=0.0001)$.

\section{Conclusions :}

These data confirm that the impulse for the use of mitral bioprosthesis for young women population has been great with an astonishing survival benefits.

Key words : Cardiac valve prostheses, Mitral valve replacement, Biological valve, Mechanical valve

\section{Introduction:}

The ultimate goal of mitral valve surgery in younger women is to extend life expectancy and improve quality of life. Mitral valve replacement prosthesis in the middle-aged women is a difficult choice between the lifelong anticoagulation of a mechanical prosthesis versus a limited long-term durability of the bioprosthesis. The current trend towards reducing the women age for selection of the mitral bioprosthesis over a mechanical prosthesis lead to a dilemma for younger women decision making. ${ }^{1,2}$ However little literatures exists supporting the use of mitral bioprosthesis over mechanical prosthesis, a lot of surgeons and cardiologists are reducing the women age recommendation for mitral bioprosthesis. ${ }^{3}$

the American College of Cardiology/American Heart Association guideline advised The mitral bioprosthetic valve for sinus rhythm younger women who chose this valve type in respect to her lifestyle considerations after comprehensive conversation of anticoagulation risk of mechanical prosthesis versus necessitating of reoperation in the future of the bioprosthesis.$^{7}$

Several literatures reported that both the mitral bioprosthesis and mechanical mitral prosthesis had an equivalent postoperative survival and quality of life..$^{8,9}$

The younger women population particularly in child-bearing period worried from the mechanical mitral prosthesis because of its bothering valve sounds, necessitating of repeated medical visits and blood tests, probability of anticoagulant-related teratogencity, thrombotic and bleeding complications. ${ }^{13}$ 
The aim of this study is to compare the clinical out-comes after mitral valve bioprosthesis versus mechanical prosthesis in younger women population when mitral valve repair not feasible or unsuitable.

\section{Materials and methods:}

This study is a single-centre registration prospective non randomized evaluation of mitral bioprosthesis performed at the Cardiothoracic Department, Ain Shams University, Cairo, Egypt, between January 2010 and February 2020.Our present study approved by the local ethical committee in accordance with Helsinki Declaration on clinical research involving human being. All patients included in our study gave written informed consent before the surgical procedures. All women patients undergoing Mitral Valve Replacement at our center from January 2010 to February 2020 included in our study. Our present study also included patients had Mitral Valve Replacement with atrial fibrillation ablation (AF) and tricuspid valve repair or replacement. Age, sinus rhythm and lifestyle consider as a recommendation for mitral valve bioprosthesis. Women want to become pregnant, with oral anticoagulant contraindication, with poor anticoagulant compliance considered for mitral bioprosthesis even if they $<60$ years old. Our exclusion criteria were infective endocarditis, prior cardiac operation, cardiogenic shock, emergency operation, and concomitant coronary artery bypass graft or aortic valve replacement. All our patients with Mitral valve bioprosthesis take oral acetylsalicylic acid (ASA) $150 \mathrm{mg}$ daily and those with Mitral valve mechanical prosthesis and/or AF take daily oral anticoagulant regularly.

All patients underwent basic preoperative demographic data (age, BMI, NYHA classification, previous cardiological interventions, cardiovascular risking factor, Comorbidities, Euroscore, echocardiography [Transthoracic Echocardiogram (TTE) - Transoesophageal Echocardiogram (TEE)], clinical data and laboratory data evaluation. Patient's preoperative and postoperative echocardiographic data [EF, LVEDD (mm), LVESD $(\mathrm{mm})$, Left atrium ( $\mathrm{mm})$, Right atrium $(\mathrm{mm})$, Mitral opening $(\mathrm{mm})$, and mitral valve pathologies] recorded. Intra-operative TEE data, as well as, the postoperative TTE data at one-week post-operative and upon clinical follow-up recorded. ${ }^{18,20}$ The relevant medical or surgical history registers. Patient's operative Variables (surgical approach [conventional or minimal invasive], Cross clamp time, Bypass time, type and size of mitral prostheses, Concomitant AF ablation and Tricuspid procedures) recorded. Patient's postoperative Variables (Ventilation time ,ICU stay, Hospital stay, pleural effusion, Pericardial tamponade, Wound infection, Arrhythmia [AV block - AF], Pneumothorax Pneumonia, Stroke, Acute renal failure, Temporary dialysis, Neurological complication, Pacemaker implantation, 30-days cardiac and non cardiac death) registered.

\section{Statistical data analyses:}

Our study continuous data presented as means \pm standard deviations (SDs) for the normally distributed variables or as median and interquartile range (IQR) for the non-normally distributed variables. The descriptive analysis for the categorical data was expressed as an absolute values and frequencies (\%).Comparative analysis for Mitral bioprosthesis and Mitral mechanical prostheses groups were achieved using Fisher's exact test or Chi-square test for categorical variables and student t test or Mann-Whitney $\mathrm{U}$ test for continuous variables as appropriate. In all cases $\mathrm{P}$ value $<0.05$ considered statistically significant. All Statistical tests were calculated using IBM SPSS 20 (IBM, USA).

\section{Results:}

Between January 2010 and February 2020, 556 female patients underwent mitral valve surgery at our center, including $64 \%(355 / 556)$ of female patients had mitral valve replacement and $36 \%(201 / 556)$ of female patients had mitral valve repair. 174 female patients of them had mitral valve replacement with mitral bioprosthesis $(\mathrm{Mb})$ and 181 female patients of them had mitral valve replacement with mitral mechanical $(\mathrm{Mm})$ prosthesis. Overall, $75 \%(266 / 355)$ of patients included in present study were female in child-bearing period. $77 \%$ ( $134 / 174)$ of Mb patients group were female in child-bearing period and $73 \%(132 / 181)$ of $\mathrm{Mm}$ patients group were female in child-bearing period. The majority of patients had Rheumatic mitral valve disease $74 \%$ (263/355) with sever MR grade [?] V 68.5\% ( 243/355) and Annular dilatation $62 \%$ $(220 / 355) .36 \%(128 / 355)$ of all patient had atrial fibrillation. Preoperative Patient demographics, mitral valve pathology and echocardiographic data compared between both groups and were statistically non- 
significant.( Table 1) of all patients mitral valve replacement $49 \%$ received Mb (Group I) and $51 \%$ received $\mathrm{Mm}$ (Group II). The operative characteristics of both groups presented in (table 2). However, the operative parameters show no statistical significant difference between the two groups, there was a significant shorter cardiopulmonary bypass time in Mb group in comparison to Mm group $(p=0.0001)$. We observe significant statistical difference in the postoperative ICU and clinical follow-up course between the both groups with excellent outcomes within patients of Mb group. (Table 3) Hospital and ICU stay was significant shorter in $\mathrm{Mb}$ group in comparison to Mm group $(p=<0.0001)$. Postoperative NYHA class status show much improvement in $\mathrm{Mb}$ group in comparison to $\mathrm{Mm}$ group $(p=0.04)$. Noteworthy there was a higher frequency for mitral valve re-replacement in Mm group in comparison to Mb group (3.7\% vs. $0.6 \% ; p=0.02$ ). The postoperative complication of mitral valve replacement included wound infection, pericardial tamponade, AF, Pneumothorax, pneumonia and acute renal failure showed non significant differences between groups. The Immediate operative death $(0.6 \%)$ was only seen in Mm group. After 10 years follow-up, we found a remarkable significant low post-operative transvalvular pressure gradient of $\mathrm{Mb}$ group in comparison to $\mathrm{Mm}$ group with $p=<0.0001$, moreover, the vena contracta was a significant more wide in patients of Mb group in comparison to $\mathrm{Mm} \operatorname{group}(p=0.006)$.

Overall, at 30 days postoperative follow-up, $3 / 355$ (0.8\%) of all patient had mitral valve replacement died, one of them in Mb group 1/174 (0.6\%) and 2/181 (1.2\%) in Mm group. The most common postoperative complication of mitral valve replacement was AF 68/355(19\%) of all patients.36/355 (10\%) of all patients experienced pneumonia, 5/355(1.4\%) wound infection, 28/355 (7.9\%) pericardial tamponade, 13/355 (3.7\%) Pneumothorax, 9/355 (2.5\%) pleural effusion and 14/355 (3.9\%) stroke. While, 34/355(9.6\%) of all patients had Acute renal failure (ARF), only 10/355(2.8\%) of them need Temporary haemodialysis. However,29/355( $8.2 \%)$ of all patient suffered of A-V block grade III, 27/355 (7.6\% ) of all patients had Pacemaker implantation. However, Re-exploration for postoperative bleeding occurred in $22 / 355(6.2 \%)$ of all patients, it was significantly higher in Mm group 18/181 (10\%) in comparison to 4/174 (2.4\%) in Mb group $(p=<$ 0.0001). While, abortion occurred in 32/355 (9\%) of all our women in child-bearing period, it was significantly higher in Mm group 27/181 (15\%) versus 5/174 (3\%) in Mb group ( $p=0.0001)$. pregnancy freedom of anticoagulant complication occurred in $68.2 \%(242 / 355)$ of all our women in child-bearing period, it was significantly higher in Mb group 141/174 (81\%) in comparison to 101/181 (56\%) in Mm group $(p=<0.0001)$. (Table 4) The postoperative complications distribution and survival rate over the follow-up period of 10 years presented in Kaplan-Meier curve. In the overall postoperative clinical follow-up period of 10 years, our present study recorded that $\mathrm{Mb}$ patients group had a significant better survival rate in comparison to $\mathrm{Mm}$ patients group. Survival rates were $92.2 \%$ for Mb group and $69 \%$ for Mm group $(p=0.01)$. The Survival rate of $\mathrm{Mb}$ group was statistically significant better than $\mathrm{Mm}$ group was. (Figure 1) However, the 95\% Confidence Interval of Cox hazards survival regression ratio was statistical significant between Mb group and Mm group [0.1926 (0.0759to 0.4889) with $p=0.0001]$, the adjustment 95\% Confidence Interval Cox hazards survival regression ratio for preoperative variables (age, logistic Euroscore-I ,MR grade 4) was no statistical significant differences between both group [0.5581(0.3254 -0. 0.9581) with $p=0.059670]$.

\section{Discussion:}

This single-centre study gives a current delineation of the safety and efficacy of the two mitral valve prostheses hypotheses. Event rate of mitral valve re-replacement appears high among Mm group and we consider this the result of poor compliance of the oral anticoagulant among young women due to their desire to be pregnant. Furthermore our study confirmed that the remarkable high success rate of $\mathrm{Mb}$ in younger women, while $\mathrm{Mm}$ in younger women associated with an increased risk of the postoperative bleeding. However, Both types of mitral prosthesis have advantages and disadvantages, the peculiar patient characteristics must be consider during the decision-making for mitral valve prosthesis type. ${ }^{14,15}$

Recent report demonstrated that $\mathrm{Mb}$ has superior antithrombotic properties and longer durability, while $\mathrm{Mm}$ associated with thromboembolism and bleeding events. ${ }^{8,} 16$ Our study proved that the use of oral anticoagulation among the Mm young women associated with a remarkable risk of postoperative bleeding, abortion and increase frequency of the pregnancy related complication of the anticoagulation, moreover, 
there is a considerable survival benefits for younger women patients received $\mathrm{Mb}$.

Kaplan-Meier curve for the long-term survival of our study at 10 years follow-up of mitral valve replacement among young women demonstrated that there was a higher significant survival with Mb (92.2\%) in comparison to $\mathrm{Mm}(69 \%)$, in spite of survival being equivalent for both group in the first three years of the study. Our 95\% Confidence Interval Cox regression analysis declared that while there was a significant survival difference between $\mathrm{Mb}$ and $\mathrm{Mm}$ [hazards ratio $=0.1926(0.0759$ to 0.4889$)$ with $p=0.0001$ ], after adjustment for preoperative base line variables (age, logistic Euroscore-I ,MR grade 4) there was no significant difference between both group [hazards ratio $=0.5581(0.3254-0$. 0.9581) with $p=0.059670]$. The peculiar patient characteristics including child-bearing period, the frequency of medical visits and blood tests, the possibility of anticoagulant-related thrombotic and bleeding events and teratogencity particularly in pregnant women influence the choice of mitral valve prosthesis type in young women. The rate of Comorbidities, such as atrial fibrillation, renal failure, Obesity, Diabetes mellitus and operative factors did not show any statistically significant difference between both groups. In the future, Mb implantation in young women will get the benefit of the trans-catheter valve-in-valve technique when structural valve deterioration occurs and that will decrease the risk of reoperation, Furthermore, newer Mb with long durability may also make Mb more point attractive for the young women population from both the patient's perspective and medical care.

Our study match the Society of Thoracic Surgeons database report from 2000 to 2007 which declared that the women age receiving mitral bioprosthesis was significantly reduced and the mitral mechanical valve implantation decreased from $68 \%$ to $37 \%$ among young women. ${ }^{11}$ Our present study aggree with Joseph Woo and associate reported that the young age was not a risk factor for the structural deterioration of the mitral valve bioprosthesis. Moreover, the mitral bioprosthesis Long-term postoperative follow-up for 20 years demonstrated that The freedom from the reoperation of the new mitral bioprosthesis due to structural valve deterioration was 91,76 and $50 \%$ at 10,15 and 20 years respectively. ${ }^{10}$

A recent confirmed data shows that only $62 \%$ of young women received mechanical mitral prosthesis on oral anticoagulation found within the acceptable international normalized ratio range. ${ }^{4,5,19}$ Patricia and associate proved that the mitral bioprosthesis structural deterioration is unclear and believed to be a result of calcium and lipids accumulation over the valve surface. So, the complete saline rinse of the mitral bioprosthesis more than once before surgery leads to a considerable decrease in structural deterioration frequency. ${ }^{6}$ However, in the past, no one can deny the limited durability consequence of the old mitral bioprosthesis, the new generation of the mitral bioprosthesis had an excellent long- term durability and performance for $>25$ years. $^{11}$

The factors contributed to the impulse use of mitral valve bioprostheses in young female patients at childbearing period are the classic surgical recommendation of the life time anticoagulation for the mechanical mitral prosthesis for young women patients, the lower risk of reoperation with the new generation of the mitral bioprostheses, the future valve in valve technique and young female patients' decision to not accept the activity constraints associated with anticoagulants. ${ }^{13,17}$

Limitations of our study:

Our study limitations that necessitate considerations are; our present study is non randomized prospective study and the patient population is definitely limited, for all the previously bring up limitations, our study need verification with a larger prospective randomized study sample.

\section{Conclusions:}

The impulse for the use of mitral bioprosthesis for young women population has been great. The recorded 10-year survival rate is higher in $\mathrm{Mb}$ than $\mathrm{Mm}$ in young women patients, but the adjusted Cox regression analysis demonstrated that there is no significant difference between the two groups, therefore, the valve selection decision making in young women is important for patient survival.

There is no funding or affiliations. 
There are no conflicts of interest.

\section{References:}

1. Frater RW, Furlong P, Cosgrove DM, Okies JE, Colburn LQ, Katz AS, Lowe NL, Ryba EA. Long-term durability and patient functional status of the Carpentier-Edwards Perimount pericardial bioprosthesis in the aortic position. J Heart Valve Dis 1998; 7(1):48-53.

2. G. Masters, Thierry G. Mesana and Marc Ruel Alexander Kulik, Pierre Bedard, B-Khanh Lam, Fraser D. Rubens, Paul J. Hendry, Roy G. Masters, Thierry G. Mesana and Marc Ruel. Mechanical versus bioprosthetic valve replacement in middle-aged patients. Eur J Cardiothorac Surg 2006;30:485-491 DOI: $10.1016 /$ j.ejcts.2006.06.013

3. Joanna Chikwe, Yuting P. Chiang, Natalia N. Egorova, Shinobu Itagaki, David H. Adams. Survival and Outcomes Following Bioprosthetic vs Mechanical Mitral Valve Replacement in Patients Aged 50 to 69 Years. JAMA. 2015; 313(14):1435-1442. doi:10.1001/jama.2015.3164

4. Maggie N Tillquist, Thomas M Maddox. Cardiac crossroads: deciding between mechanical or bioprosthetic heart valve replacement. Patient Preference and Adherence 2011:5 91-99

5. Rahimtoola SH. Choice of prosthetic heart valve in adults. Am Coll Cardiol. 2010; 55:2413-2426.

6. Patricia M Applegate, W. Douglas Boyd, Richard L. Applegate, Hong Liu. Is it the time to reconsider the choice of valves for cardiac surgery: mechanical or bioprosthetic? J Biomed Res 2017 ;31(5): 373-376 doi:10.7555/JBR.31.20170027

7. Nishimura RA, Otto CM, Bonow RO, et al. 2014 AHA/ACC guideline for the management of patients with valvular heart disease: executive summary: a report of the American College of Cardiology/American Heart Association Task Force on Practice Guidelines. Circulation. 2014; 129:2440-92.

8. Yin Wang, Si Chen, Jiawei Shi, Geng Li and Nianguo Dong. Mid- to long-term outcome comparison of the Medtronic Hancock II and bi-leaflet mechanical aortic valve replacement in patients younger than 60 years of age: a propensity-matched analysis. Interactive CardioVascular and Thoracic Surgery (2015) 1-7 doi:10.1093/icvts/ivv347

9. Cunanan CM, Cabiling CM, Dinh TT, Shen SH, Tran-Hata P, Rutledge JH et al. Tissue characterization and calcification potential of commercial bioprosthetic heart valves. Ann Thorac Surg 2001; $71: 417-21$.

10. Y. Joseph Woo and Christina L Greene.Prosthetic valve choice in middle-aged patients:guidelines and other guiding principles. Eur J Cardiothorac Surg 2016;49:1468-1469

11. James S.Gammie, Shubin Sheng, Bartley P. Griffith, Eric D. Peterson, J.Scott Rankin, Sean M. O'Brien, James M.Brown. Trends in Mitral Valve Surgery in the United States: Results from The Society of Thoracic Surgeons Adult Cardiac Database. Ann Thorac Surg 2009; 87:1431-9.

12. Carlo Valfrè,Paolo Ius, Giuseppe Minniti,Loris Salvador,Tomaso Bottio,Francesco Cesari, Giulio Rizzoli, Gino Gerosa.The fate of Hancock II porcine valve recipients 25 years after implant. Eur J Cardiothorac Surg 2010;38:141-146

13. Tsuyoshi Kaneko, Sary Aranki, Quratulain Javed, Siobhan McGurk, Prem Shekar, Michael Davidson, Lawrence Cohn. Mechanical versus bioprosthetic mitral valve replacement in patients $<65$ years old. J Thorac Cardiovasc Surg 2014;147:117-26)

14. Tillquist MN, Maddox TM. Cardiac crossroads: deciding between mechanical or bioprosthetic heart valve replacement. Patient Prefer Adherence. 2011; 5:91-9.

15. Fino C, Iacovoni A, Pibarot P, Pepper JR, Ferrero P, Merlo M, et al. Exercise hemodynamic and functional capacity after mitral valve replacement in patients with ischemic mitral regurgitation: a comparison of mechanical versus biological prostheses. Circ Heart Fail. 2018; 11:e004056.

16. Iung B, Rodes-Cabau J. The optimal management of anti-thrombotic therapy after valve replacement: certainties and uncertainties. Eur Heart J.2014; 35:2942-9.

17. Jaya Batra ,Shinobu Itagaki, Natalia N Egorova, Joanna Chikwe .Outcomes and Long-term Effects of Pregnancy in Women With Biologic and Mechanical Valve Prostheses. Am J Cardiol .2018;122 (10): 1738-1744

18. Chiara Sordelli, Sergio Severino, Luigi Ascione, Pasquale Coppolino, Pio Caso. Echocardiographic 
Assessment of Heart Valve Prostheses. J Cardiovasc Echogr. 2014; 24(4): 103-113.

19. Hui C, Lili M, Libin C, Rui Z, Fang G, Ling G, Jianping Z. Changes in coagulation and hemodynamics during pregnancy: a prospective longitudinal study of 58 cases. Arch Gynecol Obstet 2012, 285:12311236.

20. Javier G. Castillo, Jorge Solı's, A’ ngel Gonza' lez-Pinto, David H. Adams. Surgical Echocardiography of the Mitral Valve. Rev Esp Cardiol. 2011; 64(12):1169-1181

Table 1: preoperative demographic base line data

\begin{tabular}{|c|c|c|c|}
\hline & Mitral bioprosthesis & $\begin{array}{l}\text { Mitral mechanical } \\
\text { prostheses }\end{array}$ & $\mathrm{P}$ value \\
\hline Age (years) & $47.6 \pm 9.9$ & $46.5 \pm 10$ & 0.30 \\
\hline Childbearing period & $134 / 174(77 \%)$ & $132 / 181(73 \%)$ & 0.39 \\
\hline $\operatorname{BMI}\left(\mathrm{Kg} / \mathrm{m}^{2}\right)$ & $26.2 \pm 1.6$ & $26.5 \pm 1.8$ & 0.098 \\
\hline Hypertension (\%) & $77 / 174(44 \%)$ & $76 / 181(42 \%)$ & 0.70 \\
\hline Diabetes mellitus (\%) & $47 / 174(27 \%)$ & $45 / 181(24.8 \%)$ & 0.64 \\
\hline Obesity $(\%)$ & $44 / 174(25.3 \%)$ & $46 / 181(25.4 \%)$ & 0.93 \\
\hline $\begin{array}{l}\text { Familiar history of } \\
\text { cardiac surgery }(\%)\end{array}$ & $19 / 174(11 \%)$ & $27 / 181(15 \%)$ & 0.26 \\
\hline $\begin{array}{l}\text { Past history of } \\
\text { cardiology intervention } \\
(\%)\end{array}$ & $9 / 174(5 \%)$ & $11 / 181(6 \%)$ & 0.68 \\
\hline $\begin{array}{l}\text { History of previous } \\
\text { mitral valve repair (\%) }\end{array}$ & $5 / 174(3 \%)$ & $7 / 181(3.7 \%)$ & 0.61 \\
\hline Renal failure (\%) & $6 / 174(3.6 \%)$ & $6 / 181(3.3 \%)$ & 0.87 \\
\hline $\begin{array}{l}\text { Cerebrovascular } \\
\text { accident }(\%)\end{array}$ & $4 / 174(2.4 \%)$ & $3 / 181(1.7 \%)$ & 0.83 \\
\hline Chronic lung disease (\%) & $25 / 174(14.4 \%)$ & $28 / 181(15.5 \%)$ & 0.75 \\
\hline $\begin{array}{l}\text { Peripheral vascular } \\
\text { disease }(\%)\end{array}$ & $26 / 174(15 \%)$ & $29 / 181(16 \%)$ & 0.79 \\
\hline $\mathrm{AF}(\%)$ & $61 / 174(35 \%)$ & $67 / 181(37 \%)$ & 0.69 \\
\hline Euroscore & $5.6 \pm 1.4$ & $5.9 \pm 2.3$ & 0.14 \\
\hline NYHA class & $3.86 \pm 0.34$ & $3.88 \pm 0.31$ & 0.56 \\
\hline Echocardiographic data & Echocardiographic data & Echocardiographic data & Echocardiographic data \\
\hline $\begin{array}{l}\text { : } \\
\text { Mitral valve pathology }\end{array}$ & $:$ & $:$ & $:$ \\
\hline MS $(\%)$ & $57 / 174(32.8 \%)$ & $59 / 181(32.6 \%)$ & 0.97 \\
\hline $\begin{array}{l}\text { Sever MR }(>\text { grade } 4) \\
(\%)\end{array}$ & $118 / 174(68 \%)$ & $125 / 181(69 \%)$ & 0.84 \\
\hline Rheumatic (\%) & $127 / 174(73 \%)$ & $136 / 181(75.1 \%)$ & 0.67 \\
\hline Degenerative (\%) & $31 / 174(18 \%)$ & $29 / 181(16 \%)$ & 0.62 \\
\hline Endocarditis (\%) & $16 / 174(9 \%)$ & $14 / 181(7.7 \%)$ & 0.73 \\
\hline $\mathrm{EF}(\%)$ & $50 \pm 10$ & $52 \pm 12$ & 0.09 \\
\hline LVEDD (cm) & $5.1 \pm 0.7$ & $5 \pm 0.5$ & 0.12 \\
\hline $\operatorname{LVESD}(\mathrm{cm})$ & $3.6 \pm 0.4$ & $3.7 \pm 0.6$ & 0.07 \\
\hline $\mathrm{LA}(\mathrm{cm})$ & $4.3 \pm 0.5$ & $4.4 \pm 0.7$ & 0.12 \\
\hline $\mathrm{RA}(\mathrm{cm})$ & $4.6(3.9-5.2)$ & $4.8(4-5.5)$ & 0.32 \\
\hline PAP (mmHg) & $43 \pm 7$ & $44 \pm 9$ & 0.24 \\
\hline Annular dilatation & $106 / 174(61.1 \%)$ & $114 / 181(63 \%)$ & 0.71 \\
\hline $\begin{array}{l}\text { Annular or subvalvular } \\
\text { Calcification }\end{array}$ & $49 / 174(28 \%)$ & $58 / 181(32 \%)$ & 0.41 \\
\hline
\end{tabular}


BMI: Body Mass Index; AF: Atrial Fibrillation; Euroscore: predicted the risk of cardiac operations with low risk: $<2$ points; moderate risk: 3-5 points; high risk: $>6$ points; NYHA: New York Heart Association; MS: Mitral stenosis; MR: Mitral Regurgitation; EF: Ejection Fraction; LVEDD: left ventricular end diastolic diameter; LVESD; left ventricular end systolic diameter; LA: Left Atrium; RA: Right Atrium; PAP: Pulmonary Artery Pressure.

Table 2: operative data

\begin{tabular}{llll}
\hline & Mitral bioprosthesis $(\mathrm{Mb})$ & $\begin{array}{l}\text { Mitral mechanical } \\
\text { prostheses }(\mathrm{Mm})\end{array}$ & P value \\
\hline CPB time (min) & $105 \pm 15.7$ & $115 \pm 18.2$ & 0.0001 \\
ACC time (min) & $73 \pm 12$ & $75 \pm 13$ & 0.13 \\
Mitral Valve size : & Mitral Valve size : & Mitral Valve size : & Mitral Valve size : \\
$25 \mathrm{~mm}(\%)$ & $24 / 174(14 \%)$ & $27 / 181(15 \%)$ & 0.79 \\
$27 \mathrm{~mm}(\%)$ & $79 / 174(45 \%)$ & $89 / 181(49 \%)$ & 0.45 \\
$29 \mathrm{~mm}(\%)$ & $66 / 174(38 \%)$ & $58 / 181(32 \%)$ & 0.24 \\
$31 \mathrm{~mm}(\%)$ & $5 / 174(3 \%)$ & $7 / 181(4 \%)$ & 0.61 \\
Concomitant AF & $61 / 174(35 \%)$ & $67 / 181(37 \%)$ & 0.69 \\
$\begin{array}{l}\text { ablation (\%) } \\
\text { Tricuspid valve repair }\end{array}$ & $54 / 174(31 \%)$ & $53 / 181(29 \%)$ & 0.68 \\
$\begin{array}{l}\text { (\%) } \\
\text { Tricuspid valve }\end{array}$ & $9 / 174(5 \%)$ & $13 / 181(7.1 \%)$ & 0.43 \\
replacement (\%) & & & \\
\hline
\end{tabular}

ACC: aortic cross-clamping; CPB: cardiopulmonary bypass; AF: Atrial Fibrillation

Table 3: postoperative follow-up data

\begin{tabular}{lllc}
\hline & Mitral bioprosthesis $(\mathrm{Mb})$ & Mitral mechanical prostheses (Mm) & P value \\
\hline Ventilation time (hour) & $13.8(9-18)$ & $14.3(8-20)$ & 0.17 \\
ICU stay (hour) & $38.2 \pm 15.7$ & $46 \pm 17$ & $<0.000$ \\
Hospital stay (hour) & $11.7 \pm 2$ & $15.5 \pm 2.6$ & $<0.000$ \\
NYHA class & $1.1 \pm 0.4$ & $1.2 \pm 0.5$ & 0.04 \\
Wound infection (\%) & $2 / 174(1.2 \%)$ & $3 / 181(1.7 \%)$ & 0.82 \\
Pericardial tamponade (\%) & $11 / 174(6.5 \%)$ & $17 / 181(9.3 \%)$ & 0.33 \\
AV block grade III (\%) & $13 / 174(7.3 \%)$ & $16 / 181(8.8 \%)$ & 0.61 \\
AF (\%) & $37 / 174(21 \%)$ & $31 / 181(17 \%)$ & 0.34 \\
Pneumonia (\%) & $17 / 174(9.5 \%)$ & $19 / 181(10.5 \%)$ & 0.82 \\
Pneumothorax (\%) & $4 / 174(2.4 \%)$ & $9 / 181(5 \%)$ & 0.27 \\
Pleural effusion (\%) & $6 / 174(3.6 \%)$ & $3 / 181(1.7 \%)$ & 0.19 \\
Pacemaker implantation (\%) & $13 / 174(7.3 \%)$ & $14 / 181(7.7 \%)$ & 0.94 \\
Acute renal failure (\%) & $16 / 174(9 \%)$ & $18 / 181(10 \%)$ & 0.75 \\
Temporary dialysis (\%) & $3 / 174(2 \%)$ & $7 / 181(3.7 \%)$ & 0.27 \\
Stroke (\%) & $6 / 174(3.6 \%)$ & $8 / 181(4.4 \%)$ & 0.66 \\
Re-exploration for postoperative bleeding $(\%)$ & $4 / 174(2.4 \%)$ & $18 / 181(10 \%)$ & $<0.000$ \\
The Immediate operative Death & 0 & $1 / 181(0.6 \%)$ & 0.29 \\
30-day Death (\%) & $1 / 174(0.6 \%)$ & $2 / 181(1.2 \%)$ & 0.29 \\
30-day Cardiac Death (\%) & 0 & 0 & 0.01 \\
30-day non Cardiac Death (\%) & $1 / 174(0.6 \%)$ & $2 / 181(1.2 \%)$ & 0.007
\end{tabular}




\begin{tabular}{lllc}
\hline & Mitral bioprosthesis $(\mathrm{Mb})$ & Mitral mechanical prostheses $(\mathrm{Mm})$ & $\mathrm{P}$ value \\
\hline 10 years non Cardiac Death & $4 / 174(2.4 \%)$ & $6 / 181(3.3 \%)$ & 0.58 \\
Mitral re-replacement & $1 / 174(0.6 \%)$ & $7 / 181(3.7 \%)$ & 0.02 \\
Abortion & $5 / 174(3 \%)$ & $27 / 181(15 \%)$ & 0.0001 \\
pregnancy free of anticoagulant complication & $141 / 174(81 \%)$ & $101 / 181(56 \%)$ & $<0.0001$ \\
Echocardiographic data : & & & 0.44 \\
EF (\%) & $53 \pm 11$ & $52 \pm 13$ & 0.05 \\
LVED (cm) & $5.1 \pm 0.3$ & $5 \pm 0.6$ & 0.08 \\
LVESD (cm) & $3.7 \pm 0.6$ & $3.8 \pm 0.5$ & 0.07 \\
LA (cm) & $4.3 \pm 0.4$ & $4.2 \pm 0.6$ & 0.09 \\
RA (cm) & $4.7(4.2-5.2)$ & $4.8(4.2-5.4)$ & $<0.0001$ \\
Transprosthetic pressure gradient (mmHg) & $7 \pm 3$ & $15 \pm 4$ & 0.08 \\
PAP (mmHg) & $43 \pm 5$ & $42 \pm 6$ & 0.006 \\
width of the vena contracta $(\mathrm{cm})$ & $0.67 \pm 0.08$ & $0.64 \pm 0.12$ & \\
\hline
\end{tabular}

Variable described as patients applicable/patients with available information with percentage in brackets; ICU: Intensive Care Unit; NYHA: New York Heart Association; AV block: Atrio-Ventricular block; AF: Atrial Fibrillation; EF: Ejection Fraction; LVEDD: left ventricular end diastolic diameter; LVESD; left ventricular end systolic diameter; LA: Left Atrium; RA: Right Atrium; PAP: Pulmonary Artery Pressure.

Table 4: 10 year's outcomes

\begin{tabular}{llll}
\hline & Mitral bioprosthesis $(\mathrm{Mb})$ & Mitral mechanical prostheses $(\mathrm{Mm})$ & OR 95\% \\
\hline Re-exploration for postoperative bleeding (\%) & $4 / 174(3.2 \%)$ & $18 / 181(10 \%)$ & 0.2052( \\
10 years Death (\%) & $6 / 174(3.5 \%)$ & $18 / 181(10 \%)$ & 0.3234( \\
Re-operation (\%) & $1 / 174(0.6 \%)$ & $7 / 181(3.7 \%)$ & 0.1437( \\
Abortion & $5 / 174(3 \%)$ & $27 / 181(15 \%)$ & 0.1687( \\
pregnancy free of anticoagulant complication & $141 / 174(81 \%)$ & $101 / 181(56 \%)$ & 3.3843( \\
Pacemaker implantation (\%) & $13 / 174(7.3 \%)$ & $14 / 181(7.5 \%)$ & 0.9632( \\
Acute renal failure (\%) & $16 / 174(9 \%)$ & $18 / 181(10 \%)$ & 0.8624( \\
Temporary dialysis (\%) & $3 / 174(2 \%)$ & $7 / 181(4 \%)$ & 0.4361( \\
Stroke (\%) & $6 / 174(3.5 \%)$ & $8 / 181(4.4 \%)$ & 0.7723( \\
30-day Death (\%) & $1 / 174(0.6 \%)$ & $2 / 181(1.1 \%)$ & 0.5173( \\
Wound infection (\%) & $2 / 174(1.4 \%)$ & $3 / 181(1.7 \%)$ & 0.6899( \\
Pericardial tamponade (\%) & $11 / 174(6.5 \%)$ & $17 / 181(9.3 \%)$ & 0.6510( \\
AV block grade III (\%) & $13 / 174(7.5 \%)$ & $16 / 181(9 \%)$ & 0.8327( \\
AF (\%) & $37 / 174(21 \%)$ & $31 / 181(17 \%)$ & 1.3068( \\
Pneumonia (\%) & $17 / 174(9.5 \%)$ & $19 / 181(10.5 \%)$ & 0.9232( \\
Pneumothorax (\%) & $4 / 174(2.3 \%)$ & $9 / 181(4.7 \%)$ & 0.4497( \\
Pleural effusion (\%) & $6 / 174(3.5 \%)$ & $3 / 181(1.4 \%)$ & 2.1190( \\
\hline
\end{tabular}

Variable described as patients applicable/patients with available information with percentage in brackets. OR: Odds Ratio calculated by logistic regression; AOR: Adjusted Odds Ratio for preoperative age, logistic EuroScore-I and MR grade 4; AV block: Atrio-Ventricular block; AF: Atrial Fibrillation

Figure1: Kaplan-Meier curve for the long-term survival after mitral valve replacement. 


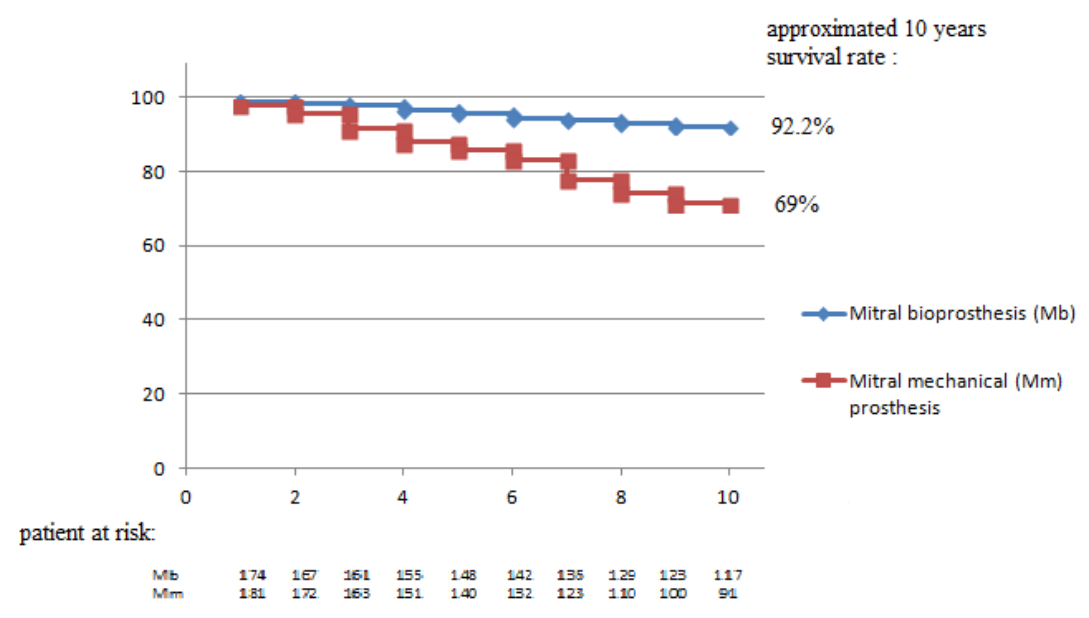

